\title{
Lignin as a Fortifier for the Synthesis of Cellulose Composite Aerogel with Strong Mechanical Performance
}

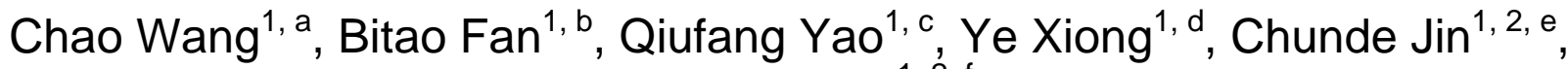 \\ Qingfeng Sun ${ }^{1,2, f}$ \\ ${ }^{1}$ School of Engineering, Zhejiang A \& F University, Lin'an 311300, PR China \\ ${ }^{2}$ Key Laboratory of Wood Science and Technology, Zhejiang Province, PR China \\ achaowangzafu@163.com, b18357178962@163.com, cyaoqiufang105@163.com, \\ dxy470849325@163.com, 'zafujincd@163.com, 'qfsun@zafu.edu.cn
}

\begin{abstract}
Keywords: Cellulose, Composite aerogels, Lignin, Mechanical performance
Abstract. The cellulose composite aerogels that were both high porosity and strong mechanical performance would have promising implications for bioengineering field; however, creating this aerogels had a challenge to choose compatible fortifier. Here we reported lignin as a reinforcement element to synthesis the cellulose composite aerogels with strong mechanical performance. Our approach - straightforwardly dissolved in ionic liquids and simply regenerated in the deionized water-causes assembly of micro-and nanoscale and even molecule level of cellulose and lignin. The resulting aerogels exhibited a strong mechanical performance. The successful synthesis of this aerogels developed a path for lignin to an advanced utilization.
\end{abstract}

\section{Introduction}

Natural sustainable resources have attracted unprecedented interest among researchers due to the rapid diminishment of oil resources and serious environmental pollution. Cellulose, the most abundant renewable natural polymer, has been one of the popular choices in current research for advance materials, because it possesses many useful features, such as hydrophilicity, biocompatibility, hydroxyl reactivity, and reasonable thermal and mechanical stabilities [1, 2]. Cellulose aerogels are one of promising candidates for various advanced applications [3] as a result of their low densities, small pore size and high-surface areas of the internal structures [4]. However, the utilization of typical cellulose aerogels was hampered by their poor mechanical performance. Therefore, there was the key that synthesis composite cellulose aerogels with a strong mechanical performance.

A typical method is hybridization with different fortifiers, such as some metallic oxide, polyurethane, poly (methyl methacrylate), and polystyrene [5-7]. In recent years, Lignin as a reinforcement of materials was reported [8]. Lignin, the abundant renewable natural polymer, is a heterogeneous and amorphous polymer of phenolic nature [9]. It is massively produced as a byproduct from papermaking and emerging cellulosic ethanol industries, and more than $98 \%$ of this material is directly poured into nearby waters or burned in an energy-recovery step [10]. So far, most of the applications of lignin still remain at a relatively low and rural level due to it not miscible with most polymers and even exhibiting deteriorated mechanical properties after mixing [11].

Herein, in this work, we employed 1-butyl-3-methylimidazolium chloride (BMIMCl), a common RTILs, as the solvent of simultaneous dissolution for the synthesis the cellulose composite aerogel with the lignin as a reinforcement element. The wet gels were produced by simple solution-gelation chemical approach. After solvent exchange processing and further freeze drying, the obtained aerogels possessed good mechanical performance.

\section{Materials and methods}

Materials. Cellulose and lignin (Sigma-Aldrich Co. 1td, USA) were analytical grade and used as received. The BMIMCl was purchased from Shanghai Boylechem Co. Itd (Shanghai, China). Other chemicals were used as received without further purification. 


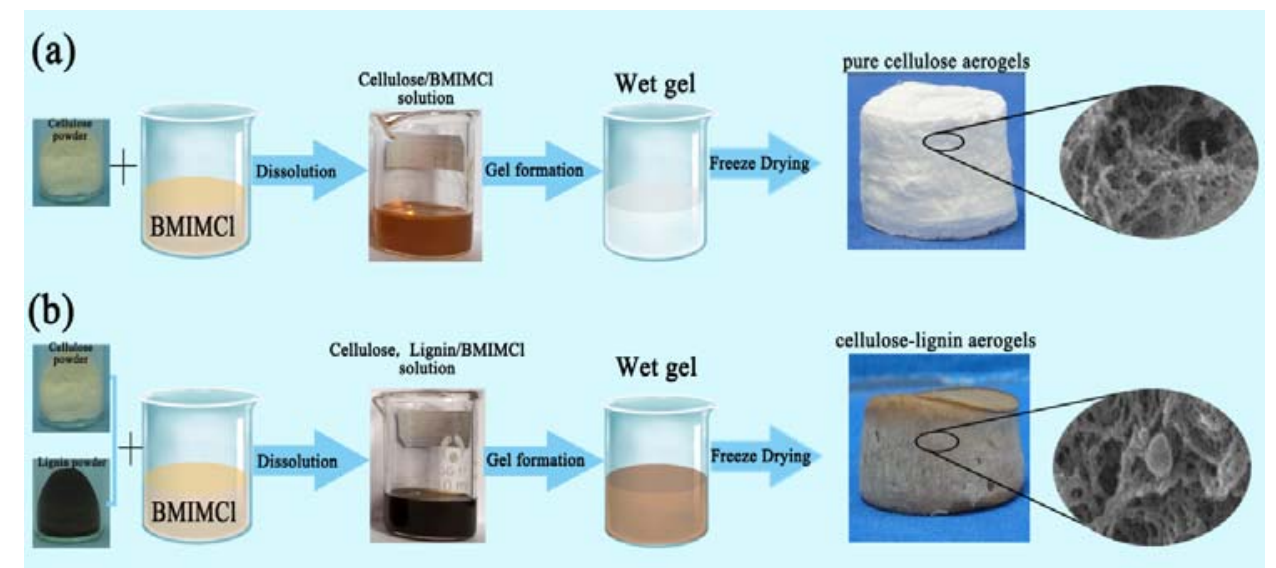

Fig. 1. Schematic presentation of samples preparation

Synthesis of samples. The detailed fabrication process is described in Fig. 1 . Cellulose powders $(0.2$ $\mathrm{g})$ and/or the same weight of lignin powders $(0.2 \mathrm{~g})$ were mixed with $9.8 \mathrm{~g}$ of BMIMCl in a $25 \mathrm{~mL}$ beaker. The beakers were immersed in an oil bath at $85^{\circ} \mathrm{C}$ via magnetic stirring for $3 \mathrm{~h}$ to form a homogeneous solution. The homogeneous viscous solutions were transferred to $10 \mathrm{~mL}$ beakers to obtain a defined thickness. Then, all samples were immersed in the first regeneration bath with the deionized water. The bath was replenished at least thrice until $\mathrm{Cl}^{-}$was not detected using $\mathrm{AgNO}_{3}$ solution. Next the gels respectively were immersed with absolute ethanol and tert-butyl alcohol to obtain more homogeneous cellular structure. The immersion time in each bath, which was replenished at least thrice, was longer than $6 \mathrm{~h}$. After undergoing several exchange processes, the wet gels (tert-butyl alcohol) were freezed drying by refrigerator at $-50{ }^{\circ} \mathrm{C}$ for more than $48 \mathrm{~h}$.

Characterizations. The morphology was observed for samples sputter-coated with gold by using a Quanta $^{\text {TM }}-250$ field-emission SEM (FEI, USA) at an accelerating voltage of $20 \mathrm{kV}$. Nitrogen adsorption-desorption isotherms were measured at $77 \mathrm{~K}$ with a Micromeritics ASAP 2020 surface area and porosity analyzer (Micromeritics instrument Ltd., USA). Compression tests were carried out on a CMT-6104 electromechanical universal testing machine (MTS systems (China) Co. ltd). During the test, the samples with thickness of $5 \mathrm{~mm}$ were compressed with a speed of $1 \mathrm{~mm} \cdot \mathrm{min}^{-1}$ until reaching a maximum load of $1 \mathrm{kN}$. Three specimens were measured for each sample.

The density of the sample $\left(\rho_{S}\right)$ was calculated from its weight $(\mathrm{g})$ and volume $\left(\mathrm{cm}^{3}\right)$. The porosity of the sample $(\mathrm{P})$ was calculated by the following Eq. 1, where $\left(\rho_{\mathrm{C}}\right)$ and $\left(\rho_{\mathrm{L}}\right)$ respectively is the density of bulk cellulose $\left(1.52 \mathrm{~g} / \mathrm{cm}^{3}\right)$ and lignin $\left(1.30 \mathrm{~g} / \mathrm{cm}^{3}\right), \mathrm{n}$ is the quality percentage of cellulose for lignin.

$$
P=\left(1-\frac{\rho_{S}}{n \rho_{C}+(1-n) \rho_{L}}\right) \times 100
$$

\section{Results and discussion}

The pure cellulose aerogels were synthesized for comparison (Fig. 1). Macroscopically, the samples possess good integrity and well-defined shapes without any cracks. Obviously, color of composite aerogels is suited well with that of lignin, and it is not worth noting that there has been a slight shriveling composite aerogels with of lignin. On the macroscopic shape depend on the microscopic morphology. 

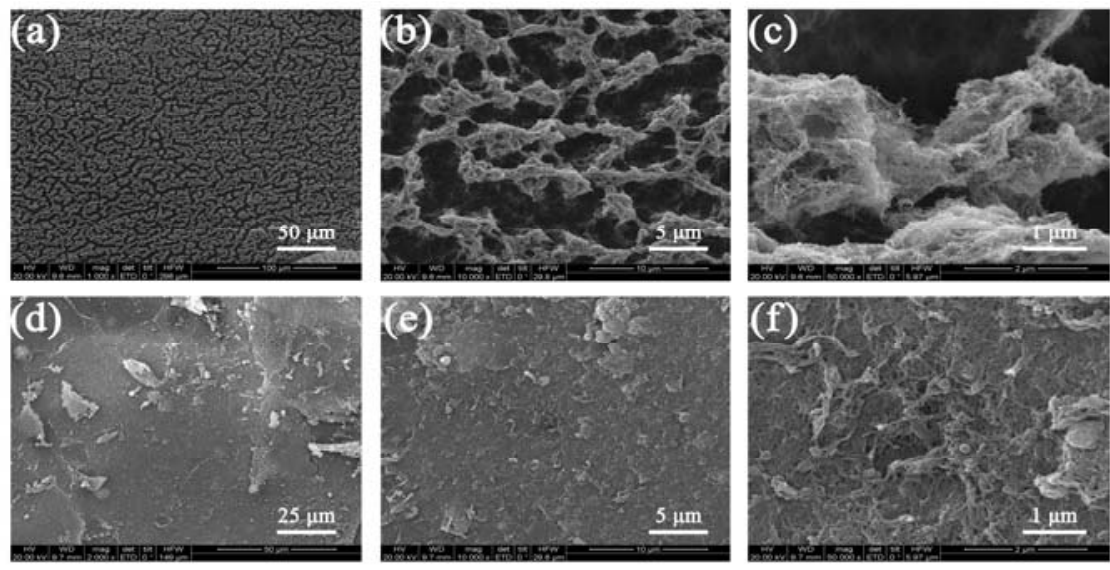

Fig. 2 SEM images of samples: pure cellulose aerogels (a, b, c), lignin-cellulose aerogels (d, e, f)

The distinct morphologies were shown by scanning electron microscopy (SEM) images for samples. Fig. 2a and c, show irregularly shaped microscale and nanoscale pores of pure cellulose aerogels at the different magnification. Under high magnification $(\times 50,000)$, it is shown that aggregated and interconnected cellulose nanofibers (CNF) make up the thick walls of the macroscopic. Fig. 2 d, low magnification $(\times 2,000)$, shows denser morphologies, consistent with the slight shrink of macro shape and the increase of density (Table 1). The pores were smaller and even cannot be found because of adding lignin in cross-linked network of cellulose. Under high magnification $(\times 50,000)$, the images clearly show nanoscale pores enclosed by CNF, and lignin was relative uniformly filled in this pores (Fig. 2f).

Table 1. Physical properties of the samples.

\begin{tabular}{ccccccc}
\hline Sample & $\begin{array}{c}\text { density } \\
{\left[\mathrm{g} \mathrm{cm}^{-3}\right]}\end{array}$ & $\begin{array}{c}\text { porosit } \\
\text { [\%] }\end{array}$ & $\begin{array}{c}\mathrm{S}_{\mathrm{BET}} \\
{\left[\mathrm{m}^{2} \mathrm{~g}^{-1}\right]}\end{array}$ & $\begin{array}{c}\text { pore volume }^{\mathrm{a}} \\
{\left[\mathrm{cm}^{3} \mathrm{~g}^{-1}\right]}\end{array}$ & $\begin{array}{c}\text { pore size }^{\text {a }} \\
{[\mathrm{nm}]}\end{array}$ & $\begin{array}{c}\text { Young's } \\
\text { modulus } \\
{[\mathrm{Mpa}]}\end{array}$ \\
\hline $\begin{array}{c}\text { Pure cellulose } \\
\text { aerogels } \\
\begin{array}{c}\text { Lignin-cellulose } \\
\text { aerogels }\end{array}\end{array}$ & 0.021 & 98.4 & 258.7 & 1.25 & 17.8 & 0.86 \\
\hline
\end{tabular}

${ }^{\mathrm{a}}$ Obtained from desorption isotherms by BJH method.

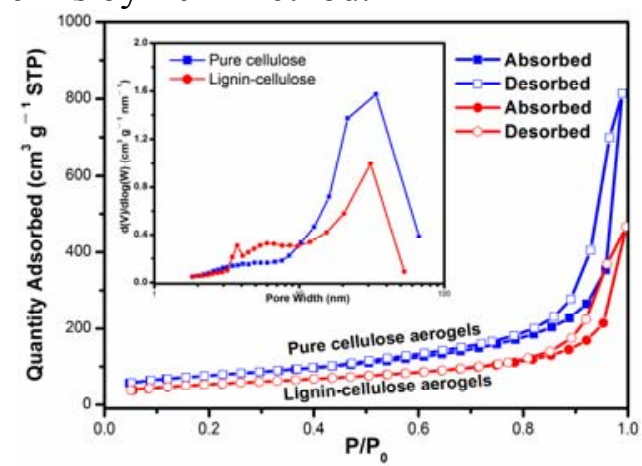

Fig. 3 Nitrogen adsorption and desorption isotherms. The inset shows the BJH desorption pore distribution of the samples.

The specific surface area of the samples $\left(\mathrm{S}_{\mathrm{BET}}\right)$, calculated from the Brunauer-Emmett-Teller (BET) equation, were respectively $258.7 \mathrm{~m}^{2} \mathrm{~g}^{-1}$ for pure cellulose aerogels and $190.8 \mathrm{~m}^{2} \mathrm{~g}^{-1}$ for lignin-cellulose samples (Table 1). The nitrogen adsorption-desorption isotherms have a distinct hysteresis loops observed in Fig. 3, and are of type IV according to IUPAC classification. A smaller hysteresis loop of composite aerogels also could be observed in Fig. 3. They correspond to the presence of mesoporous structures. The pore width distribution curves from the desorption branch by the Barrett-Joyner-Halenda (BJH) method of samples were plotted the inset of Fig. 3. It is shows that the samples contain pores ranging from $1 \mathrm{~nm}$ to $100 \mathrm{~nm}$, and the average pore diameter were 17.8 and $12.7 \mathrm{~nm}$ and total pore volumes 1.25 and $0.68 \mathrm{~cm}^{3} \mathrm{~g}^{-1}$, respectively (Table 1). In the range of 10-30 
$\mathrm{nm}$, a predominant peak can be observed in pore diameter distribution of the samples. Furthermore, that of lignin-cellulose aerogels can be found a well sub-peak about $4 \mathrm{~nm}$, which also agree with their SEM images at high magnification.
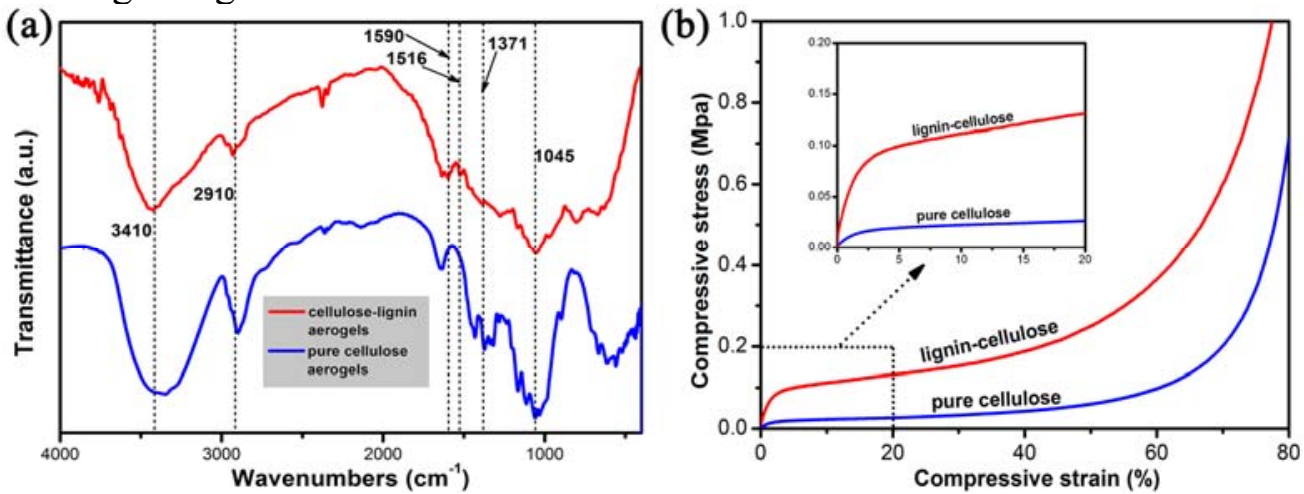

Fig. 4. (a) Comparison of FTIR spectra of samples. (b) Compressive stress-strain curves for the samples (5 mm thick).

As shown in Fig. 4a, the samples exhibits the characteristic bands of cellulose at 2910, 1371 and $1045 \mathrm{~cm}^{-1}$ corresponding to the $\mathrm{C}-\mathrm{H}$ stretching and deformation modes, the $\mathrm{C}-\mathrm{O}-\mathrm{C}$ symmetric and antisymmetric stretching and the $\mathrm{C}-\mathrm{O}$ stretching modes [12]. After this mixed lignin, the unchanged spectral signature of cellulose reveals a good stability of the samples. Besides of the FTIR spectra of $\mathrm{S}-0$, the others can be observed the appearance of different characteristic peaks: one relative to the aromatic skeletal vibration and $\mathrm{C}=\mathrm{O}$ stretching located at $1590 \mathrm{~cm}^{-1}$, evidently, resulting from the lignin. Fig. 4b shows the compressive stress-strain curves of all samples with thickness of $5 \mathrm{~mm}$ up to $80 \%$ strain. The samples display a small linear elastic region about $2 \%$. The sample mixed lignin, showing dramatic increase with strain, had a Young's modulus up to 4.0 MPa, which is 5 times above the pure cellulose aerogels as compare. But beyond the yield point, all curves showed a plateau where the stress rises tardily with growing compressive strain, indicating that the porous structure in samples collapsed increasingly under the compressive load. Toward higher strains, all samples showed a soaring increase in stress due to the densification of samples after complete collapse of the porous structure. Both samples did not breakage until the force transducer of the testing machine reached its setting limit, although the deformation of specimens was irreversible. The advantage was lack in common inorganic counterparts. The stress-strain curves suggested that mixing the lignin in cellulose can improve compression resistance.

\section{Conclusions}

Cellulose composite aerogels of lignin as a fortifier were successfully prepared by dissolving cellulose and lignin powders in BMIMCl, regenerating in the deionized water and freezing drying process. The unmodified lignin, applications of relatively low and rural level, was reconstructed into 3D lamellar porous cellulose aerogels. We envision that the samples will open up numerous opportunities for a range of applications in dampers, heat insulators, sieves, absorbents and tissue engineering scaffolds.

\section{Acknowledgements}

The work was financially supported by Zhejiang Provincial Natural Science Foundation of China (No. LZ14C160001), Public Projects of Zhejiang Province (No. 2015C32014), Scientific Research Foundation of Zhejiang A\&F University (Grant No. 2014FR077), and Fund for Innovative Research Team of Forestry Engineering Discipline (101-206001000713). 


\section{References}

[1] L. Yu, K. Dean and L. Li: Prog. polym. Sci. 31 576-602 (2006)

[2] I. Siró and D. Plackett: Cellulose 17 459-494 (2010)

[3] F. Liebner, E. Haimer, M. Wendland, M. A. Neouze, K. Schlufter, P. Miethe, T. Heinze, A. Potthas and T. Rosenau: Macromol. Biosci. 10 349-352 (2010)

[4] F. Liebner, A. Potthast, T. Rosenau, E. Haimer and M. Wendland: Holzforschung 62 129-135 (2008)

[5] N. Leventis: Accounts chem. Res. 40 874-884 (2007)

[6] N. Leventis, A. Sadekar, N. Chandrasekaran and C. Sotiriou-Leventis: 2010 Chem. Mater. 22 2790-2803

[7] H. Zou, S. Wu and J. Shen: Chem. Rev. 108 3893-3957 (2008)

[8] K. Akato, C. D. Tran, J. Chen and A. K. Naskar: ACS Sustain. Chem. Eng. 3 3070-3076 (2015)

[9] A. M. Boudet: Trends plant sci. 3 67-71 (1998)

[10] W. Thielemans, E. Can, S. Morye and R. Wool: J. Appl. Polym. Sci. 83 323-331 (2002)

[11]S. Kubo and J. F. Kadla: Biomacromolecules 4 561-567 (2003)

[12]R. H. Marchessault and C. Y. Liang: J. Polym. Sci. 43 71-84 (1960) 